\title{
Centres urbains secondaires et commercialisation des produits vivriers au Togo
}

\section{Frédéric Lançon}

\section{Résumé}

Les centres urbains secondaires sont des points d'interconnexion entre la collecte, le commerce de gros et la vente au détail des produits vivriers. La consommation de leurs habitants diversifie les débouchés et permet ainsi d'assouplir la gestion des flux commerciaux et de diminuer les risques inhérents à cette activité. C'est dans ce cadre urbain que se sont développées des corporations de commerçantes et les spécificités de la vie urbaine poussent certaines femmes à s'introduire dans la profession afin d'assurer l'approvisionnement de leur famille en vivrier. Le renforcement de la sécurité alimentaire du Togo est donc en partie lié à l'avenir de ces villes secondaires.

\begin{abstract}
Secondary cities are located at the intersection of assembling, wholesale distribution and retailing in foodstuffs. Therefore, they act as buffer between the different stages of the distribution process. Their inhabitants'consumption increases the level of the final demand and multiply the number of marketing channels which facilitates the management of the whole system and lowers the trading risk. Trade guilds are closely linked with those cities, and women, who are in charge of supplying the family with food, often get into this business to achieve this goal and not only to earn money. Then, Togo's food policy has to take into account the future of small and medium size cities.
\end{abstract}

\section{Citer ce document / Cite this document :}

Lançon Frédéric. Centres urbains secondaires et commercialisation des produits vivriers au Togo. In: Économie rurale. N¹90, 1989. pp. 33-40;

doi : https://doi.org/10.3406/ecoru.1989.3962

https://www.persee.fr/doc/ecoru_0013-0559_1989_num_190_1_3962

Fichier pdf généré le 08/05/2018 


\section{CENTRES URBAINS SECONDAIRES ET COMMERCIALISATION DES PRODUITS VIVRIERS AU TOGO \\ Frédéric LANÇON *}

\section{Résumé :}

Les centres urbains secondaires sont des points d'interconnexion entre la collecte, le commerce de gros et la vente au détail des produits vivriers. La consommation de leurs habitants diversifie les débouchés et permet ainsi d'assouplir la gestion des flux commerciaux et de diminuer les risques inhérents à cette activité. C'est dans ce cadre urbain que se sont développées des corporations de commerçantes et les spécificités de la vie urbaine poussent certaines femmes à s'introduire dans la profession afin d'assurer l'approvisionnement de leur famille en vivrier. Le renforcement de la sécurité alimentaire du Togo est donc en partie lié à l'avenir de ces villes secondaires.

\section{Summary :}

\section{MEDIUM SIZE CITIES AND FOOD CROP MARKETING IN TOGO}

Secondary cities are located at the intersection of assembling, wholesale distribution and retailing in foodstuffs. Therefore, they act as buffer between the different stages of the distribution process. Their inhabitants'consumption increases the level of the final demand and multiply the number of marketing channels which facilitates the management of the whole system and lowers the trading risk. Trade guilds are closely linked with those cities, and women, who are in charge of supplying the family with food, often get into this business to achieve this goal and not only to earn money. Then, Togo's food policy has to take into account the future of small and medium size cities.

Comme dans de nombreux autres pays d'Afrique subsaharienne, la forte croissance de l'urbanisation au Togo (1) ne nous conduit-elle pas à associer la place des villes dans la filière agro- alimentaire à la seule fonction de consommation ? Cette conception n'est-elle pas aussi renforcée par la réduction du fait urbain aux seules capitales? Certes la part de ces dernières dans la population urbaine totale ne cesse de croître (2), mais si macrocéphalie il y a, nous ne devons pas pour autant négliger l'autre face de l'urbanisation que sont les centres urbains secondaires. Les résultats de deux enquêtes (3) réalisées dans la Région des Plateaux montrent que ceux-ci occupent des positions stratégiques dans l'organisation des filières à régulation marchande - pour reprendre la typologie établie par Ph. Hugon (Altersial, 1986, p. 30) - par laquelle transite la plus grande part de l'approvisionnement en produits vivriers des urbains togolais (4).

Après avoir exposé en quoi leur spécificité urbaine participe à la structuration et à la régulation des flux de produits vivriers, du producteur au consommateur, nous insisterons sur le rôle important de ces centres urbains secondaires dans le développement des différentes catégories d'intermédiaires.

\section{LE SYSTÈME DE COMMERCIALISATION}

Le système d'approvisionnement de la capitale s'appuie sur trois des cinq régions administratives du Togo. Il est schématisé sur la carte 1 (5). Les flèches et les cercles ne symbolisent pas des volumes de produits commercialisés, mais le pourcentage des intermédiaires qui ont déclaré

\footnotetext{
- CeRED/LAREA, Université de Paris X - Nanterre

1. Sur la période 1970-1981 les taux de croissance démographique de la population urbaine et de la population totale du Togo ont été respectivement estimés à 4,4\% et 2,85\% (CEE/CDC, 1983 pp. 359-366).

2. La part de Lomé dans la population urbaine totale peut étre estimbe $46 \%$ en 1971 et à $55 \%$ en 1981, selon des calculs effectués a partir de CEE/CDC. op. cit., p. 364

3. Ces deux enquêtes, l'une sur les ventes des producteurs, l'autre sur les circuits de commercialisation et les pratiques des intermediaires font partie d'un programme de recherche, dirigée par l'ORSTOM (J.P. Minvielle, F. Ayebouya, F. Lançon et $Y$. Tossou, 1985).
}

acheter ou vendre sur telle ou telle place de marché. Environ $58 \%$ des flux sont originaires des places de marché de la Région Maritime qui sont situées en moyenne à trente kilomètres de la capitale. Selon le même critère, les places de marché de la Région des Plateaux, distantes en moyenne
4. Nous limiterons l'emploi du terme * produits vivriers * aux produits suivants : maïs, sorgho, riz, sésame, niébé, arachide, manioc (tubercules et farine), igname et taro. D'aprés une étude de la SOTED la proportion de maïs commercialisée par l'Office national de commercialisation des céréales (Togograin) n'aurait jamais dépassé $15 \%$ (1 \% en 1981) (Soted, 1981, p. 33). On peut estimer qu'en calories, la part des importations de blé, de riz et de maiss ont representé entre $6 \%$ et $10 \%$ de la production locale sur la période $1977-1980$; calculs effectués à partir du bilan établi par le Ministere de la Coopération $(1984$, p. 5) et de l'étude de la Soted (op. cit. p. 8 et annexe 2/5).

5. Cartes établies à partir d'informations recueillies dans N. Assouline et A. Guillaume-Gentil, 1986, pp. 29.30. 
de 144 kilomètres, sont à l'origine de $34 \%$ des flux de vivriers qui convergent vers Lomé. Enfin, les $8 \%$ restant proviennent de la Région Centrale et de la Région de la Kara (distance moyenne des places de marché : 351 kilomètres). Les circuits de longues distances (distances supérieures à 100 kilomètres) sont en grande majorité des cir- cuits de gros ; par conséquent la part de la Région Maritime doit être minorée au profit des deux autres, et surtout de la Région des Plateaux, puisque les régions septentrionales du pays sont essentiellement excédentaires en igname (Soted, op. cit., cartes 1 à 7).

Carte 1. - Origines des flux de vivrier qui approvisionnent la capitale

Légendes :

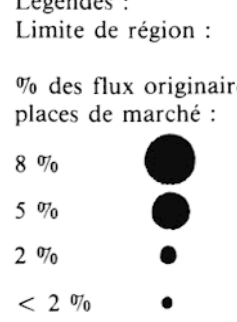

$<2 \%$

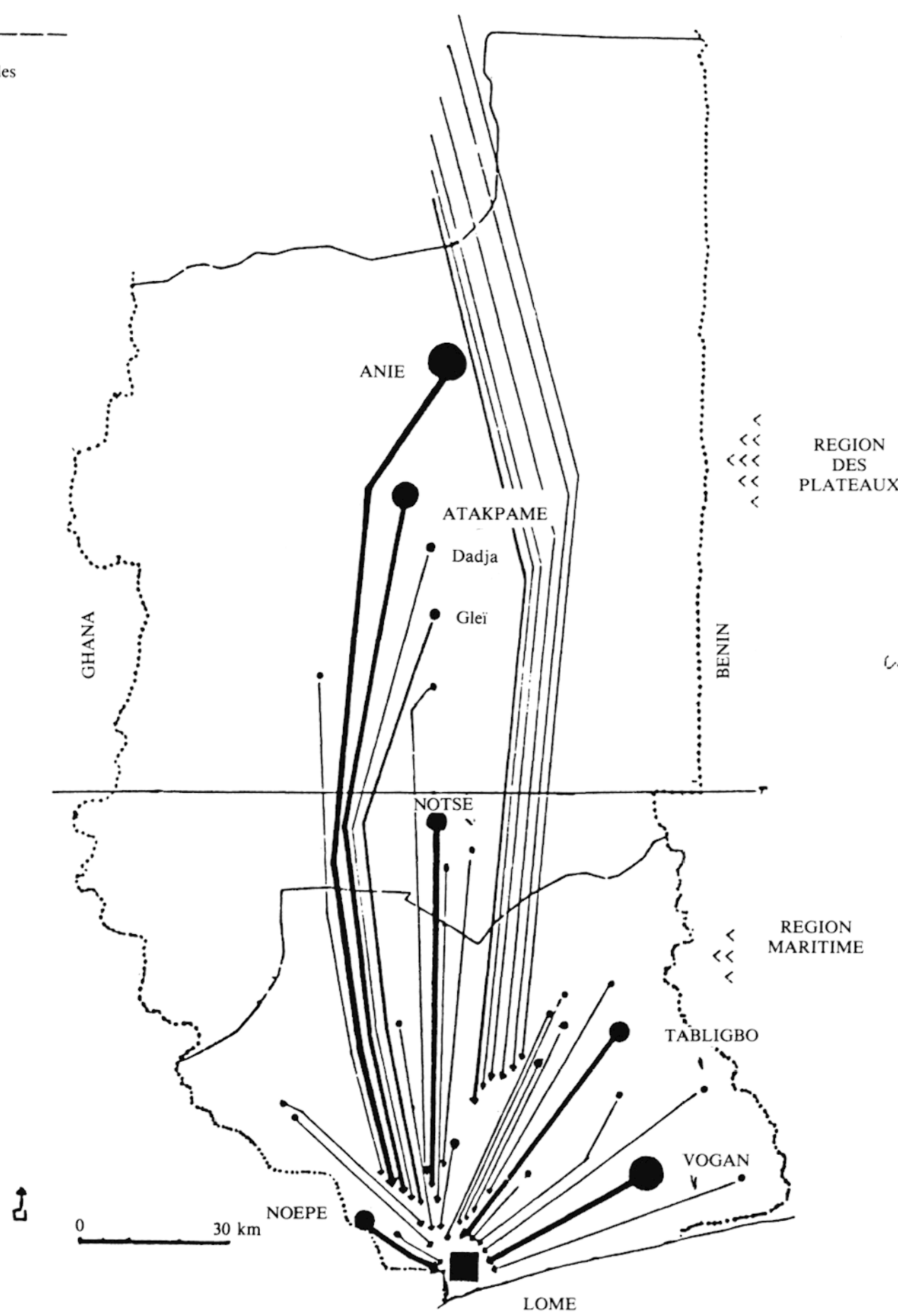

Nous focaliserons donc notre étude sur la Région des Plateaux. Reliée à Lomé par deux axes routiers de bonne qualité, deux lignes de chemin de fer, vétustes mais toujours exploitées, cette région est, et sera de plus en plus, un élément capital du système d'approvisionnement de la capitale. En effet, la Région Maritime reste le premier fournisseur de Lomé, mais son éco-système a de plus en plus de mal à endiguer une utilisation de moins en moins extensive de sols fragiles.

On peut découper le système de commercialisation en quatre niveaux dans lesquels on regroupera les flux qui n'appartiennent pas au même circuit, mais qui se situent au même stade de commercialisation. 
Le premier niveau (carte 2) englobe l'ensemble des opérations de ventes conduites par les producteurs. En règle générale c'est la femme du producteur qui se charge de cette tâche. La distance moyenne parcourue est de huit kilomètres pour l'ensemble des exploitations, elle varie de zéro à trente kilomètres suivant la localisation des villages par rapport aux axes routiers et aux principales places de marché. Une seule exploitation assure le transport de sa production sur une distance de plus de 50 kilomètres. Les quantités mises en vente dépassent rarement la trentaine de kilos, sauf dans la périphérie des places de marché les plus importantes où quelques véhicules assurent le transport des produits vivriers entre les villages et le marché. Ce niveau inclut également les ventes directes entre exploitants et consommateurs ruraux. Suivant les zones d'investigations entre $30 \%$ et $15 \%$ des producteurs déclarent pratiquer ce type de vente.

Le second niveau (carte 3) rassemble les flux qui convergent vers les pôles commerciaux les plus importants. Leurs longueurs varient en moyenne, suivant les pôles commerciaux, de 12 à 38 kilomètres. C'est sur ce niveau qu'opèrent les collectrices agissant pour le compte des grossistes, mais aussi des commerçantes que nous qualifierons, faute de terme plus approprié, « d'indépendantes ». Ce sont des intermédiaires qui assurent l'ensemble des différentes phases de la commercialisation, depuis l'achat aux producteurs jusqu'à la vente finale aux consommateurs. Certaines parmi elles remplissent conjointement le rôle de collectrice. Les volumes des transactions sont en règle générale limités à une dizaine de sacs. C'est entre ce deuxième niveau et le troisième que s'opère la scission entre des circuits courts à un seul intermédiaire et les circuits longs où interviennent plusieurs opérateurs.

Le troisième niveau (carte 4) est en effet celui du commerce de gros. Il comprend les circuits qui assurent le transit des produits vivriers à l'échelle régionale et nationale. Ils relient des places de marché distantes de plusieurs dizaines et même centaines de kilomètres. Ce sont ceux qui figurent aussi sur la carte 1 , à l'exception d'une partie des flux originaires de la Région Maritime,qui appartiennent au deuxième niveau. C'est par définition le niveau des transactions de volumes importants : plusieurs dizaines de sacs de céréales ou de légumineuses, des camions remplis de tubercules d'igname. Ces circuits sont aux mains des grossistes ; certaines d'entre elles, nous les appellerons « petites grossistes ", ajoutent à cette activité la revente au détail d'une partic de la marchandise grâce à une maind'œuvre familiale.

Enfin le quatrième niveau (carte 5) est celui de la redistribution (ou de la vente au détail) assurée par des inter- médiaires spécialisés ou non (détaillantes, commerçantes indépendantes, petites grossistes). Ils opèrent soit dans un centre urbain (Lomé, Kpalimé), soit dans le centre urbain et à sa périphérie (Badou, Notsé) sur des places de marchés situées en moyenne à moins de 20 kilomètres.

C'est au troisième niveau que s'opère le rééquilibrage de la balance alimentaire régionale et nationale. Au niveau régional on observe un rééquilibrage au profit de l'ouest. Cela traduit une forte propension à la commercialisation du vivrier des producteurs de la partie est de la Région des Plateaux (carte 1), alors que la partie occidentale est structurellement déficitaire (zone de forte densité démographique dont la culture d'exportation - le café/ cacao - s'harmonise moins bien avec les productions vivrières que celle de la partie est : le coton). Les centres secondaires situés à l'ouest, Kpalimé et Badou, ne sont que des centres de consommation ou de redistribution vers les marchés ruraux. Au niveau national, c'est à travers ces circuits que la région participe aux ravitaillements de Lomé et dans des proportions beaucoup plus faibles à celui des centres secondaires du nord (Lama Kara et Kétao).

Si l'on croise les informations des cartes 1 et 4 , on remarque que les places de marché de la Région des Plateaux qui participent au commerce de gros sont au nombre de 11 ; 9 sont des points de départ, ou « d'exportation », et 2 des points d'arrivée ou "d'importation ». Si l'on considère les flux qui convergent vers Lomé, on constate que les places d'Anié, d'Atakapmé et de Notsé sont à l'origine de $56 \%$ de ces flux (N. Assouline et A. Guillaume Gentil, op. cit., p. 30).

La place de marché d'Anié, la plus importante de la région, est particulière. En effet, parmi les cinq pôles commerciaux les plus importants (Anié, Atakapmé, Notsé, Kpalimé et Badou), c'est la seule qui ne soit pas au cœur d'un centre urbain secondaire. Cependant, les projections démographiques montrent que cette singularité risque fort de disparaître dans les dix années à venir (Y. Marguerat, « L'armature urbaine du Togo », ORSTOM, Paris, 1985, p. 147). Avec une population avoisinant les 30000 habitants, en l'an 2000, le village d'Anié deviendrait à son tour un centre secondaire au caractère urbain de plus en plus affirmé. Cet exemple montre que les synergies entre les dynamiques commerciales et urbaines maintes fois décrites (cf. les travaux de F.Braudel) peuvent être encore d'actualité dans certaines régions africaines. La combinaison d'une agriculture particulièrement dynamique (la zone nord-est de la Région des Plateaux) et d'une infrastructure de communication fiable (axe routier nord-sud) peut favoriser le développement d'un tissu urbain plus équilibré.

\section{CENTRES SECONDAIRES ET DIMINUTION DU RISQUE DE PERTE}

Anié mis à part, les pôles commerciaux les plus importants de la région sont les centres urbains secondaires. Ce sont les points d'articulation entre les niveaux 2 et 3 et les niveaux 3 et 4 . Au-delà de la séquence, collecte/concentration, circuits de gros/transport sur longue distance et déconcentration/vente au détail, qui relève d'une approche en terme de rupture de charge, volumes commercialisés et seuil de rentabilité de chacune de ces fonctions (économie d'échelle...), ces pôles sont aussi des lieux d'articulation de rythmes d'activités différents.
Prenons l'exemple d'un « circuit long », le circuit de commercialisation du maïs entre la région d'Atakapmé et la capitale. Les activités de collecte (niveau 2) se déroulent entre le lundi et le vendredi, elles suivent donc un rythme quasi-quotidien, alors que les grossistes assurent une ou au maximum deux rotations entre Atakapmé et Lomé. Enfin la vente au détail sur les places de marché de Lomé est quotidienne. L'écoulement des marchandises n'est pas continu ; le passage d'un niveau à l'autre occasionne des décalages temporels nécessitant le stockage 
Légendes :

Carte 2

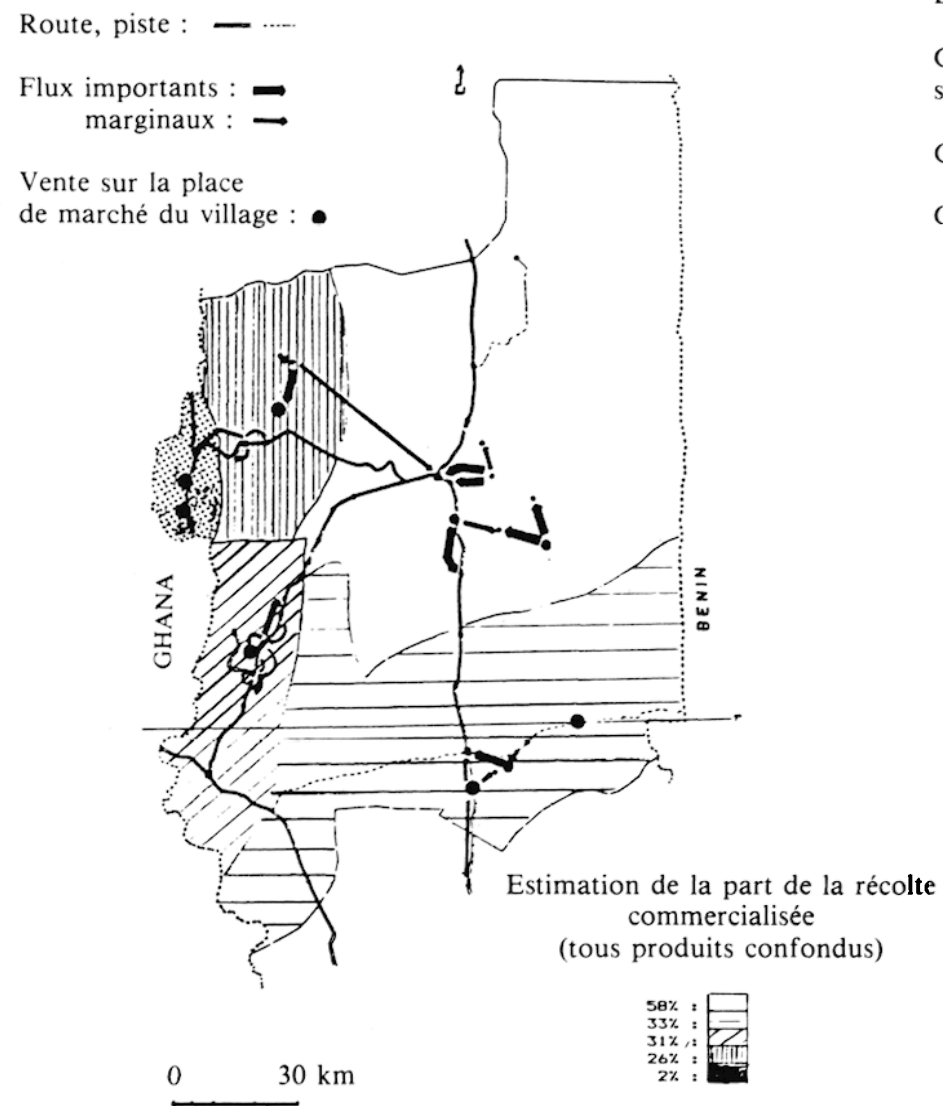

Carte 4

Légendes :

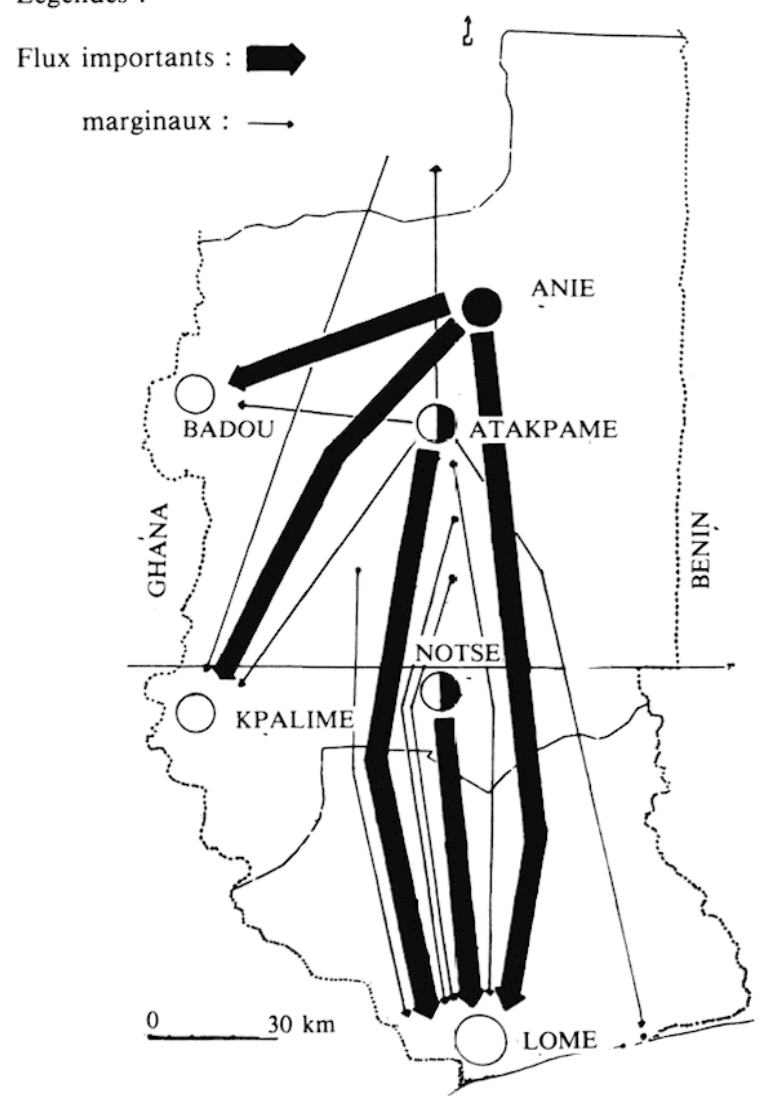

Carte 3

Légendes :

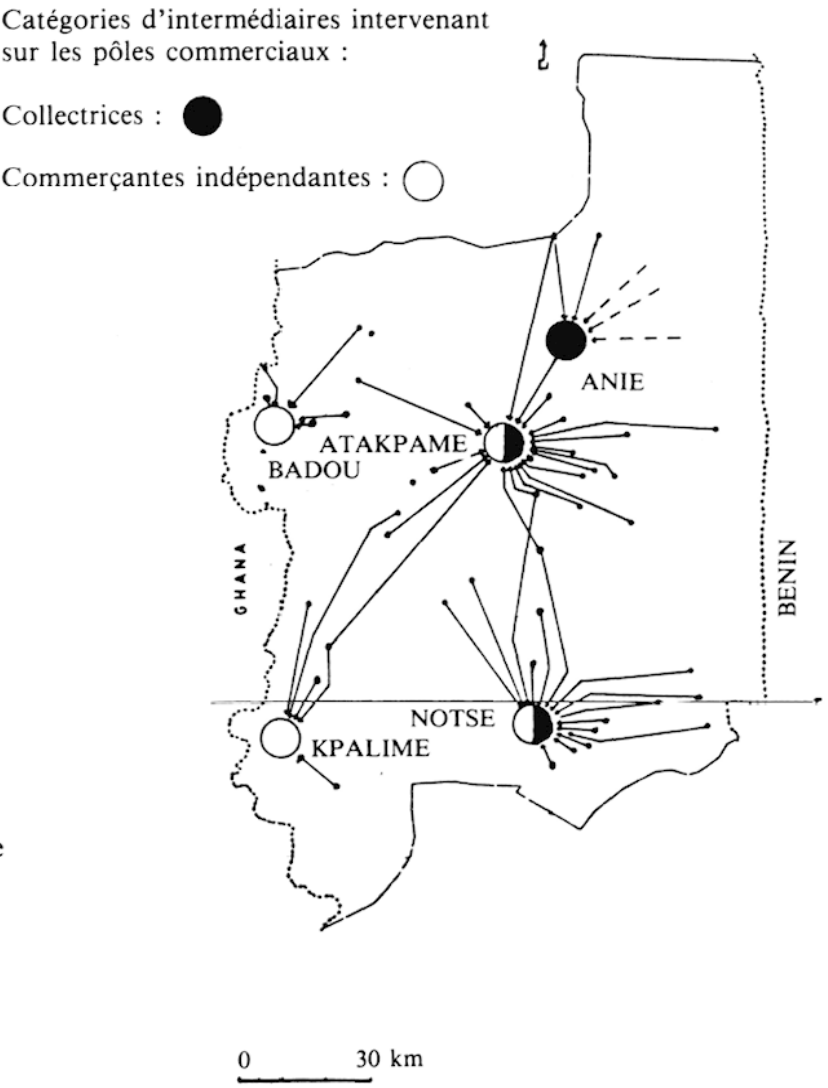

Carte 5

Légendes :

Distribution assurée par :

- Commerçantes indépendantes :

- Détaillantes :

$\theta$

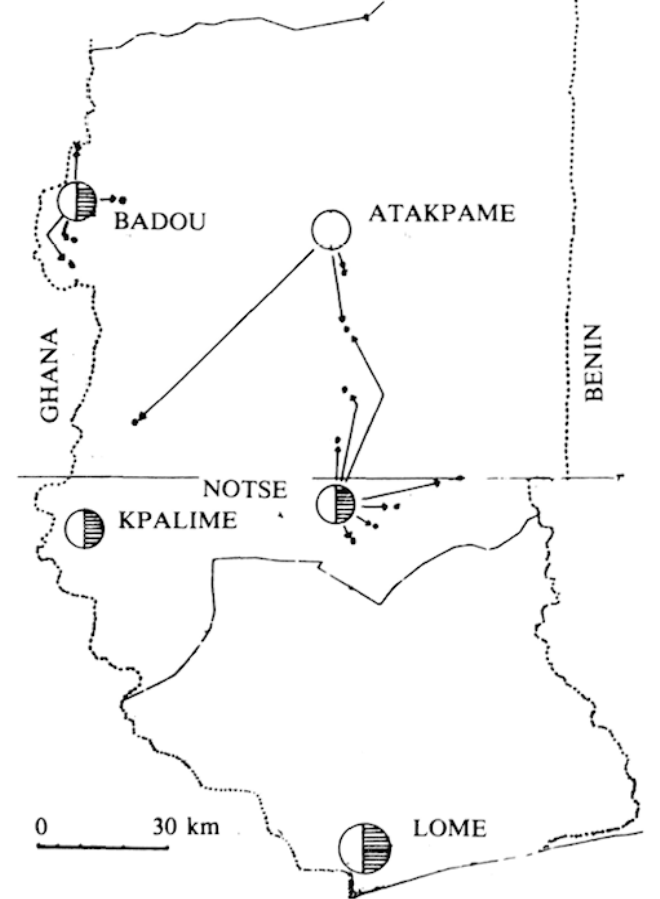


des marchandises entre deux segments du circuit. Cette capacité d'articulation entre différents rythmes d'activité est une des spécificités du monde urbain. Mais ces stocks ne jouent pas seulement un rôle dans la régulation de l'écoulement des produits entre chaque niveau ; ils ont aussi, et surtout, une fonction de régulation de l'activité par rapport aux variations de prix.

En effet, rappelons que la Région des Plateaux, et a fortiori la zone d'Atakapmé, n'est pour la capitale qu'un centre d'approvisonnement parmi d'autres. Cette multiplication des aires de productions ou d'《 exportations » favorise les variations du niveau des prix. Ainsi la mise en vente simultanée de volumes importants de marchandises au niveau 1, dans les différentes zones exportatrices, va se traduire par une baisse du prix au stade de la redistribution (liaison 3/4), qui se répercutera ensuite sur les pôles commerciaux des aires de collectes (liaison $2 / 3$ ). La circulation des produits vivriers n'est pas un processus harmonieux.Les oscillations qui scandent le déroulement des opérations de commercialisation sont la manifestation d'une structure où les décisions de vente et d'achat sont prises individuellement, de façon non coordonnée aussi bien entre chaque niveau que dans le temps et dans l'espace. Chaque intermédiaire essaye donc de mettre en œuvre des techniques qui lui permettent de se couvrir, ou de minimiser les risques de perte.

Le stockage de la marchandise dans l'attente d'une remontée du niveau des prix en est une. Cependant, elle est coûteuse, et les capitaux disponibles ne sont pas toujours suffisants pour permettre de démarrer une nouvelle rotation ; c'est surtout le cas pour les intermédiaires qui assurent la collecte. Certains intermédiaires sont donc obligés de vendre à perte, pour poursuivre leur activité. De plus cette pratique est risquée à cause des méthodes de conservation rudimentaires (pas de procédé artificiel de stockage) ;elle ne peut pas être utilisée pour les tubercules (seul le manioc est transformé en farine ce qui facilite énormément sa commercialisation).

Par conséquent, les agents du système de commercialisation utilisent d'autres mécanismes. Les centres secondaires sont un élément de certaines de ces stratégies, et partant, de la régulation de l'ensemble du système de commercialisation.

La première consiste à diversifier ses activités. Cette diversification peut se faire en multipliant les productions commercialisées. Ainsi $60 \%$ des intermédiaires travaillent avec au moins deux produits. Cette palette est plus ou moins large en fonction de la diversité des productions mises en vente par les producteurs dans chacune des zones, mais comme pour le stockage, cette stratégie est aussi en grande partie déterminée par le montant du capital disponible ; les intermédiaires de petite taille,que l'on trouve en majorité au niveau 2 et 4 peuvent donc difficilement mettre en œuvre cette pratique. Pour les mêmes raisons, le cumul du commerce des produits vivriers avec d'autres marchandises est relativement rare. $12 \%$ des intermédiaires travaillent occasionnellement avec d'autres catégories de produits vivriers (gombo, piments et plus rarement avec des fruits ou du riz importé). Une proportion encore plus faible $(3 \%)$ revend des produits non vivriers (charbon).

Les intermédiaires peuvent aussi diversifier leurs débouchés ou travailler simultanément sur des circuits courts et longs. Les consommateurs des centres urbains secondaires et des zones rurales déficitaires constituent des opportunités de répartition des risques. Pour le commerce de gros ces débouchés alternatifs restent limités par le volume de la demande et par la concurrence d'autres circuits de commercialisation, celle, par exemple, des circuits de l'igname produite dans la Région Centrale qui convergent vers Kpalimé et Badou et des circuits courts reliant ces centres de consommation à leurs périphéries. De plus, l'activité commerciale à ce niveau est caractérisée par un fort cloisonnement des circuits. Cela se conçoit très bien dans la mesure où les relations avec les agents chargés de la vente au détail, qui sont situés en aval, sont capitales pour la bonne marche du commerce de gros. Par conséquent, les grossistes résident en règle générale dans les pôles commerciaux situés à la fin des "circuits longs " (liaison 3/4); il leur est donc quasiment impossible de travailler en même temps sur deux centres de consommation: Par ailleurs, elles possèdent généralement un capital suffisant pour se couvrir en diversifiant leurs achats.

Par contre, la diversification des débouchés est un atout important pour les intermédiaires chargés de la collecte, et qui ne peuvent pas momentanément écouler, sans perdre de l'argent, leurs marchandises dans les « circuits longs ». Une partie d'entre eux se tourne vers la demande urbaine locale, afin de reconstituer une partie de leur fonds de roulement. Ils peuvent aussi parfois revendre une partie de leurs achats en milieu rural lorsque les transactions entre producteurs (niveau 1) ne couvrent pas entièrement la demande rurale. Ainsi des circuits ville $\rightarrow$ campagne peuvent aussi exister dans des zones globalement excédentaires, mais déficitaires pour un seul produit ; c'est le cas de la farine de manioc dans la zone de Notsé.

Une autre stratégie consiste au contraire à se spécialiser dans une seule spéculation et à se protéger en exerçant un droit d'exclusivité sur la commercialisation de cette production. Cette stratégie est plus fréquente sur les circuits courts que sur les circuits longs. A Atakpamé, par exemple, coexistent deux groupes d'intermédiaires : ceux qui commercialisent les céréales (maïs, sorgho), le haricot et la farine de manioc et ceux qui travaillent exclusivement avec l'igname. Cette césure est l'objet d'une surveillance attentive de la part des vendeurs d'ignames qui sont moins nombreux et dont le débouché est exclusivement local : la place de marché d'Atakpamé. Ces derniers prennent garde à ce qu'aucun intermédiaire du premier groupe (céréales) ne s'introduise dans leur circuit. Cc comportement permet d'éviter qu'une mauvaise conjoncture sur les circuits des céréales et produits associés ne se traduise par une modification brusque du rapport entre « offreurs " (producteurs) et « demandeurs » (intermédiaires) sur le circuit de l'igname au détriment des intermédiaires spécialisés dans le commerce de ce tubercule.

Ce « marché urbain » qui est captif, n'en est pas pour autant moins concurrentiel que celui du groupe des céréales ; les barrières à l'entrée sont aussi basses dans l'un que dans l'autre. Ce qui est interdit, c'est la possibilité de « tenter sa chance " sur les deux simultanément. Cette spécialisation résulte aussi de la particularité du commerce de l'igname, activité saisonnière et produit non stockable. Les autres techniques de couverture, prêts, avances en nature ou monétaires ne sont pas directement liées à la spécificité urbaine des principaux pôles commerciaux. 
Les populations d'Atakpamé (32 000 habitants), de Kpalimé (27 500 habitants) et des autres centres secondaires ne représentent bien sûr qu'une faible part de la demande potentielle en produits vivriers par rapport aux quelques 390000 habitants de la capitale. Cependant, d'une part elles facilitent la gestion des circuits longs en offrant une possibilité de repli pour les intermédiaires qui assurent la collecte ; cette opportunité est mise à contribution par $90 \%$ des agents qui assurent la collecte autour
d'Atakpamé ( $50 \%$ pour Notsé) ; d'autre part, elles représentent un débouché additionnel qui favorise un écoulement plus régulier des productions mises en vente par les producteurs, qui sont moins dépendants des circuits qui convergent vers la capitale.

Mais le rôle des centres secondaires dans le système de commercialisation ne se limite pas à ces seules fonctions. La résidence des intermédiaires en milieu urbain est un des facteurs qui détermine l'entrée dans cette profession.

\section{COMMERÇANTES ET CITADINES}

Tous les intermédiaires sont des femmes. Cette particularité relève à la fois des modes d'insertion et des motifs qui conduisent certaines femmes à s'introduire dans le système de commercialisation des produits vivriers.

Comme toute autre activité, la commercialisation des produits vivriers ne peut être entreprise qu'après avoir acquis un certain nombre de techniques. Celles-ci vont de la maîtrise de l'utilisation des multiples unités de mesure (nous avons pu en recenser 19 - bols ou cuvettes - dans la Région des Plateaux) à la connaissance des méthodes de marchandage, des avantages et des inconvénients des moyens de transport, en passant par l'aptitude à différencier les qualités des produits offerts (produits suffisamment secs, ou non, pour être stockés). Pour $42 \%$ des commerçantes interrogées l'acquisition de ces techniques de commercialisation s'est faite en aidant leurs mères (ou une proche parente) qui étaient déjà elles-mêmes commerçantes. Pour $45 \%$ des commerçantes le capital initial est aussi obtenu par le biais d'un don de la mère.

Il y a donc un phénomène d'auto-renouvellement pour une partie des agents de la commercialisation qui se succèdent dans le cadre familial. Cet ensemble de pratiques concerne en particulier les intermédiaires les plus importants que sont les grossistes et certaines commerçantes indépendantes.

Le rôle de la ville dans l'édification de cette corporation appartient essentiellement au domaine de l'héritage historique (Marguerat, op. cit. p. 17-21). En effet, en dehors des fonctions commerciales inhérentes aux centres secondaires, que nous avons énoncées ci-dessus, la localisation urbaine de ce processus de reproduction relève aussi d'un processus de perception et de construction d'une identité sociale en partie basée sur l'appartenance au monde urbain. Les seules commerçantes qui sont propriétaires de leurs habitations appartiennent à cette catégorie d'intermédiaires. Qu'il ait été acheté ou reçu en héritage, ce patrimoine est une des marques de leur enracinement dans le monde urbain.

Les entrées dans le système de commercialisation des femmes qui interviennent au niveau de la collecte ou de la vente au détail sont aussi étroitement liées à la vie en milieu urbain.

En ville, comme en milieu rural, c'est à la femme que revient la charge de la préparation des repas. Cette tâche comprend également l'achat des produits alimentaires. Bien que la production directe de culture vivrière soit une des solutions utilisées par les citadins pour assurer leur approvisionnement, elle reste peu répandue parmi les agents de la commercialisation. D'une part, seulement $15 \%$ d'entre eux possèdent une parcelle de terre et d'autre part cette production personnelle ne couvre manifestement pas la totalité des besoins familiaux en produits vivriers.

Les achats de produits vivriers demeurent donc essentiels pour l'alimentation du groupe familial. Ils vont favoriser l'entrée de certaines femmes dans les circuits de commercialisation. En effet, lorsque l'on essaye de recenser les motifs qui ont conduit ces femmes à faire du commerce avec les produits vivriers, plus du quart d'entre elles répondent explicitement que l'objectif poursuivi est de « nourrir la famille »; certaines comparent même leur position à celle de producteurs agricoles disposant en permanence de réserve alimentaire. Cet objectif est d'autant plus important que ces commerçantes sont en règle générale des mères de famille (98\% de l'échantillon). D'ailleurs l'obtention du capital initial est très souvent associé aux mariages (dot), et par la suite le mari reste une des principales sources de refinancement.

Cette relation entre les responsabilités familiales et les contraintes de la vie urbaine permet de comprendre que malgré des marges brutes, et parfois des marges nettes, négatives, certaines femmes continuent à exercer une activité commerciale, dont la rémunération reste, encore une fois, très aléatoire. D'une part, elle explique que l'on sousestime la rentabilité de l'opération si l'on n'intègre pas dans le calcul des marges les quantités achetées qui ont été directement consommées par la famille de la commerçante; ces quantités doivent être considérées comme une partie du bénéfice qui est directement consommé en nature. D'autre part, elle permet de comprendre que les pertes fréquentes ou les faillites n'entraînent pas un retrait de la commerçante du circuit de commercialisation. La participation aux circuits de collectes, même à une petite échelle, diminue le coût de l'approvisionnement de la famille en produit vivrier. Les prix de collecte étant plus bas que les prix de vente au détail sur les places de marché des centres urbains secondaires. Cette stratégie concerne principalement les intermédiaires qui travaillent au niveau 2 des circuits de commercialisation.

La combinaison de comportements à rationalité purement marchande et à rationalité " domestique » au sein des circuits accroît l'efficacité de l'ensemble du processus de commercialisation. Grâce à celle-ci, les canaux de collectes perdurent, même durant les périodes où les écarts de prix entre les zones excédentaires et déficitaires ne sont pas suffisants pour rendre cette activité financièrement rentable. 


\section{CONCLUSION}

L'observation des circuits de commercialisation des produits vivriers au Togo semble aller dans le sens des nouvelles orientations des politiques économiques qui cherchent à rendre les cultures vivrières plus attractives pour les producteurs. La complexité de l'organisation du système de commercialisation témoigne de l'avancée des rapports marchands dans les zones rurales, où la demande urbaine est largement répercutée à travers un quadrillage conséquent de cet espace en places de marché. Cependant la commercialisation des produits vivriers demeure une activité à risque.

L'existence d'un maillage urbain dense contribue à limiter cette précarité. Les centres urbains secondaires sont non seulement des points d'articulation entre des phases de commercialisation d'échelles et de rythmes différents, mais ils sont aussi des centres de consommation. Cette demande contribue à la régulation du système, soit parce qu'elle favorise la diversification des circuits, soit parce qu'elle contribue à l'abaissement des coûts de commercialisation en favorisant le maintien des activités de collectes en partie basées sur des comportements non marchands.

Ce réseau de villes est un atout pour la pérennité d'une structure de commercialisation efficace des productions vivrières locales ; à ce titre il est un élément moteur de l'économie rurale. Cependant la part de Lomé dans la répartition des activités économiques ne cesse de s'accrô̂tre. Cette concentration de la création de richesse dans la capitale restreint d'autant les flux commerciaux des produits allant de la capitale vers l'intérieur du pays. Cette dynamique peut être néfaste à moyen terme pour les circuits des produits vivriers : la diminution de l'activité économique dans les centres secondaires aura certainement des répercussions sur le système de commercialisation qui lui est étroitement lié.

Ce déséquilibre est aussi vivement ressenti par les transporteurs, qui trouvent difficilement des volumes de frêts suffisants pour revenir de la capitale vers les centres secondaires sans perdre d'argent. C'est un handicap supplémentaire qui ne peut être surmonté que par une hausse des coûts de transport au détriment du producteur, comme du consommateur.

La nouvelle répartition du revenu national au bénéfice des ruraux, (hausse des prix au producteur) est un des axes majeurs des politiques alimentaires officielles des pays d'Afrique sub-saharienne. La réussite de ce transfert de pouvoir d'achat dépend aussi de l'efficacité , en aval, des systèmes de commercialisation. L'exemple du Togo montre donc, une nouvelle fois (cf. Wertheimer M. " Les villes secondaires en Afrique : leur rôle et leurs fonctions dans le développement régional, " in : Altersal, Cered et MSA, op. cit., 1986 pp. 136-137), que ces politiques devraient aussi être accompagnées de mesures favorables à l'activité économique des centres urbains secondaires.

\section{RÉFÉRENCES BIBLIOGRAPHIQUES}

ALTERSIAL, CERED et MSA (1986). - Nourrir les villes en Afrique sub-saharienne. L'Harmattan, Paris, 1986421 p.

ASSOULINE N. et GUILLAUME-GENTIL A. (1986). - Le circuit d'approvisionnement en vivriers de la ville de Lomé. Rapport de stage. DESS, Développement. Université de Paris X-Nanterre. Centre Orstom de Lomé, 1986105 p.

CEE/CDC (1983). - Etude d'une image à long terme de l'Afrique au sud du Sahara. Annexe démographique, tome 2, sept. 1983.

MARGUERAT Y. (1985). - L'armature urbaine du Togo. Orstom, Paris, 1985166 p.

Ministère de la Coopération (1984). - Situation alimentaire du Togo. Paris, 19845 p.
MINVIELlE J.P., AYEBOUA F., LANÇON F., TOSSOU Y. (1985). - Production, commercialisation et consommation des produits vivriers dans la région des Plateaux. Togo, Document de travail $\mathbf{n}^{\circ} 1$ : présentation méthodologique, Orstom, Lomé, 1985, 61 p.

SOTED (1981). - Diagnostic du marché des produits vivriers et facteurs explicatifs du mouvement des prix au Togo. Lomé, juin 198163 p.

WERTHEIMER M. (1986). - Les villes secondaires en Afrique : leur rôle et leurs fonctions dans le développement régional. In ALTERSIAL, CERED et MSA, Nourrir les villes en Afrique sub-saharienne, l'Harmattan, Paris, 1986 pp. 136-157. 


\section{RIVISTA \\ DI ECONOMIA AGRARIA}

Anno XLIII - n. 3 - settembre 1988

STUDI DI ECONOMIA AGRARIA

POLITICA AGRARIA, SOCIOLOGIA RURALE

Ricordo di Manlio Rossi Doria, a cura di Mario Pagella, Michele De Benedictis, Giuseppe Orlando

\section{SAGGI E RICERCHE}

Giovanni Galizzi, L'agricoltura mondiale tra crisi e contraddizioni

Luciano Iacoponi, Sergio Miele, L'analisi a «Fondi e Flussi» del processo produttivo ed il caso della concimazione delle colture

GIUSEPPE D'ANGelo, Analisi delle interdipendenze tra agricoltura $e$ industria: prime indicazioni

Antonio Cordella, Antonio De Pin, Pier luigi Ruzzi, La domanda al consumo di alimenti proteici in Italia: un'analisi con dati trimestrali per il periodo 1975-85

Franco Sotte, Andrea Arzeni, Trasformazioni strutturali nell'agricoltura italiana. Un'applicazione dell'analisi multivariata ai censimenti provinciali

Mario Cardano, La definizione di una tipologia areale dell'agricoltura piemontese: un'applicazione della Cluster Analysis

Gianluca Brunori, Francesco Di Iacovo, Mara MieLE, Agricoltura biologica: aspetti tecnici, di mercato e normativi

\section{TEMI DI DISCUSSIONE}

Alessandro Corsi, Tecniche e processo produttivo: un ulteriore commento

Franco Bruni, Contabilità e tecniche amministrative nelle imprese in agricoltura: possibili contenuti di una disciplina

\section{RECENSIONI}

Chiniro Nakajima, Subjective Equilibrium Theory of the Farm Household, di G. Balestrieri

F. Lorenzoni, L. Zappella, Politiche pubbliche di sviluppo del Mezzogiorno: la nuova disciplina organica dell'intervento straordinario (Legge n. 64/86), di A. Zezza 